\title{
CONDUIRE LE CHANGEMENT: \\ LE DÉPLOIEMENT D'ACTIONS DE FORMATION EN CULTURES IMPLICITES ET EXPLICITES ; L'IMPORTANCE DES FACTEURS CULTURELS. UN CAS D'ILLUSTRATION EN EUROPE.
}

\author{
Jean-Rémi Koutchkine*
}

\section{INTRODUCTION}

Les organisations ne peuvent plus se permettre de lourdes structures pyramidales. On constate aujourd'hui un mouvement de balancier vers les structures plates et matricielles. Ces changements rapides ont obligé les personnels, par-delà les cultures et les frontières, à changer profondément leur façon de travailler, leurs relations à l'autorité et au projet, enfin, leur propre relation à l'Entreprise. La capacité à développer de nouvelles compétences, à travailler dans un environnement changeant, à prendre des risques, à travailler dans un cadre flou, de même que la capacité à créer de nouvelles formes d'organisations, à la fois globales et locales, sont les enjeux des entreprises devenues transculturelles pour survivre et se développer dans un environnement aujourd'hui métissé, mais où en même temps les particularismes locaux n'ont jamais été aussi forts. La spécificité de l'Europe apparaît alors pleinement, mosaïque de cultures qui devront faire leur des principes qui ont été pensés et mis en oeuvre dans des cultures anglo-saxonnes. La remise en cause des modes d'organisations créés aux Etats-Unis par une culture américaine telle est souvent l'enjeu d'organisations confrontées à la mondialisation des acteurs économiques.

Une organisation confrontée à une nécessité de survie, donc de changement, a mis en oeuvre une série de formations impliquant l'encadrement dans sa conduite. Comment est-il possible d'apprendre positivement plutôt que contraint? Est-il préférable de faire évoluer son personnel plutôt que de le remplacer? L'enjeu est là, il

* Université de Paris Val-de-Marne-Paris 12. 
nécessitera l'implication de tous, en particulier ceux, qui confrontés au défi interculturel, devront agir en synergies afin de permettre la renaissance de l'entreprise tant en respectant l'identité culturelle de chacun plutôt que d'accepter une monoculture nord-américaine peu adaptée à l'Europe et à ses subtiles nuances.

\subsection{Un contexte favorable}

L'organisation objet de cette observation opère dans le secteur des biens d'équipements informatiques et des services associés. Le secteur d'activité dans lequel opère cette organisation est traditionnellement empreint de la culture américaine. En effet, la naissance de l'informatique et son développement dans les dernières décennies s'est fait à l'ombre de sociétés telles que RCA (Radio Corporation of America), IBM, Honeywell ou encore General Electric. Les modes de fonctionnement de ces organisations reposent sur la promotion interne et la qualité d'entrepreneur des acteurs:

"L'américain pris au hasard doit donc être un homme ardent dans ses désirs, entreprenant, aventureux, surtout novateur"I.

Cette organisation que nous appellerons dans le cadre de cette communication CVMM est fortement empreinte de culture américaine, nous pouvons y observer des modes de fonctionnement et d'organisation américains : Organisation en Strategic Business Units, présence d'Account Managers au sein de Sales teams autorégulés, [...]. De même, le vocabulaire en usage fait référence à la culture américaine, que ce soit pour l'usage des titres en vigueur (Vice President, Branch Managers, ...) ou pour l'usage quotidien en utilisant les termes tels que FW (Fiscal Week), Rolling forecast ou billing, en vigueur dans toutes les entités. Ce cas que nous allons étudier met en évidence le poids de la culture américaine, pragmatique et tournée vers l'action.

CVMM, en Europe, est dirigée par un European Executive Committee, où les Directeurs Généraux des Filiales se rencontrent, et un Comité Fonctionnel qui régule les activités du support et communique les orientations du siège. C'est à partir de l'exposé fait par le Directeur Général de la Grande Bretagne, filiale qui traditionnellement a été le point d'ancrage de l'actionnaire américain et dont les modes de fonctionnement et de pensée étaient eux aussi grandement sous influence américaine grâce à la langue et au cadre social de référence. La Grande Bretagne, permet facilement la mise en oeuvre de décisions, "naturelles" aux EtatsUnis mais peu acceptées sur le continent: Licenciements, promotion rapide, primauté du savoir-faire sur les diplômes. Aux Etats-Unis, "salariés et cadres peuvent être licenciés du jour au lendemain, sauf s'il existe une convention collective qui prévoit un préavis - dépassant rarement deux semaines"2.

Le Directeur Général de CVMM Grande-Bretagne fit une présentation des premiers résultats d'une "Université d'Été" dont le programme était destiné à 
accompagner une mutation des modes de fonctionnement ainsi que des plans de suivi SPOTLIGHT ${ }^{3}$ prévus dès octobre. Cette présentation retint l'attention de ses homologues et du Directeur des Ressources Humaines de CVMM Europe. Elle généra une double impulsion:

- La première, des Directeurs Généraux de filiales vers leurs encadrements, afin d'être en phase avec ce qui semblait être une initiative majeure et parfaitement cohérente avec les orientations du groupe, ces orientations étant résumées dans un recueil appelé P.D.P. (Priorities Development Plan):

For performance all levers identified are exactly the same as those already identified in the PDP process. Our challenge is to accelerate the implementation of PDP4.

Les Directeurs Généraux demandèrent à leurs staffs: "Qu'avez-vous entrepris comme actions similaires?"

En effet, la plupart de ces directeurs généraux ont une culture de gestion acquise dans les business schools américaines et le cabinet de consultants qui avait produit le rapport est lui aussi nord-américain.

- La deuxième, des financiers du siège:

PDP model suggests $10 \%$ sales force surplus vs. market needs if guide line met. If Clusterisation Business Units (sectorial) are implemented, and third party channel is recruited for non strategic market segments then further $5 \%$ is possible overall $15 \%$ reduction [...]. Flattening of structure should result in additionnal savings to "other sales" (managers, secretaries etc.) should remove $15 \%$ of other sales 5 .

Ces estimations poussaient les financiers à encourager un re-engineering des forces de ventes et une réduction des effectifs par une focalisation plus grande. Leur objectif était de tendre vers une uniformisation et une centralisation plus fortes.

\subsection{Des ressources mobilisables}

Cette situation organisationnelle allait rencontrer un potentiel d'investissements prévus, des budgets destinés à des investissements dans les domaines suivants: consultance pour la mise en oeuvre de méthodes d'optimisation des canaux de distribution, réorientation de personnels et formations lourdes.

Au sein du marketing et des ressources humaines du siège, les acteurs, principalement des cadres intermédiaires, saisirent cette opportunité afin d'étendre leur champ d'influence.

Cette émulation fut rendue encore plus forte par le fait que les leviers utilisés pour conduire le changement en Grande-Bretagne étaient accessibles et ne présentaient pas de difficultés apparentes. Le programme portait en effet sur la formation au travail en équipe, la facilitation du travail de groupe et la résolution des 
problèmes liés à ces modes de fonctionnement à travers l'utilisation d'outils et de méthodes de marketing.

Nous avons donc deux entités fonctionnelles (marketing et ressources humaines) dont l'intérêt objectif est de promouvoir l'expérience de formation anglaise dans d'autres "Clusters". ${ }^{6}$ Les acteurs de ces deux entités vont naturellement travailler avec leurs réseaux habituels formés par les cadres intermédiaires de Grande-Bretagne, tant fonctionnels qu'opérationnels, ayant contribué au déploiement des formations.

Le facteur temps va contribuer à la prise en main de ce dossier stratégique par l'encadrement fonctionnel. En effet, la fin de l'année est la période pendant laquelle les opérationnels sont totalement dédiés à leurs clients, ce qui laisse potentiellement le champ aux autres acteurs pour mener des actions d'envergure.

Un facteur important a été celui de la langue. CVMM Europe utilise comme langue de travail l'anglais, ce qui rend l'ensemble des outils, supports de cours et documentations, immédiatement disponibles. Cependant, l'usage de l'anglais comme langue de travail recouvre une réalité plus complexe qui favorise le rôle des cadres intermédiaires dans la conduite des processus. Il est en effet posé comme postulat au niveau du Comité Exécutif que tout le monde parle et écrit l'anglais à CVMM Europe et dans les filiales (c'est une réalité au niveau de ceux qui dialoguent avec le siège).

L'histoire même de ce groupe avait construit une réalité fort différente et nous avions globalement la situation suivante:

Maîtrise de la langue anglaise et française.

\begin{tabular}{lll}
\hline & Anglais & Français \\
\hline Directeurs Généraux des filiales & +++ & $+/-$ \\
\hline Jeunes très haut potentiel & ++ & -- \\
\hline
\end{tabular}

En ce qui concerne les cadres intermédiaires, on pouvait constater la maîtrise linguistique suivante:

Maîtrise de l'anglais et du français par les cadres

\begin{tabular}{lll}
\hline CADRES & Anglais & Français \\
\hline Zone Scandinavie & +++ & $+/$ \\
\hline Hollande & +++ & $+/-$ \\
\hline Allemagne & ++ & $+/$ \\
\hline Europe de l'Est & ++ & $+/-$ \\
\hline Espagne & $+l$ & ++ \\
\hline Italie & $+l-$ & ++ \\
\hline Portugal & $+l-$ & +++ \\
\hline Suisse & $+l-$ & +++ \\
\hline Belgique & $+l-$ & $+++^{\top}$
\end{tabular}


Contrairement à l'idée que se faisait le Comité Exécutif, beaucoup d'acteurs parmi les cadres intermédiaires avaient été choisis parce qu'ils parlaient une langue telle que l'espagnol ou l'italien, en plus de l'anglais qui restait pour eux une langue de secours et d'écrit.

La Direction de la Formation était parfaitement consciente de ce fait et, sensibilisée aux difficultés liées aux différences culturelles, elle s'attacha tout au long du processus de déploiement à tenir compte de ce paramètre. Mais elle n'était pas seule et cette position offrait plus de contraintes que de chances de succès rapides.

Quels furent le rôle de la langue et de la culture dans la conduite du changement à travers l' exemple que nous avons retenu, et, par rapport à la culture locale, le rôle joué par les cadres intermédiaires du siège dans la mise en oeuvre de formations au changement?

\subsection{Le facteur culturel}

E. Schein, enseignant au MIT, décrit une culture comme étant "une structure faite de postulats fondamentaux inventés, découverts ou élaborés par un groupe donné quand il apprend à faire face à ses problèmes d'adaptation externes et d'intégration interne, et qui a fonctionné assez bien pour être considéré comme valide et enseigné aux nouveaux membres et tant que mode juste de perception, de pensée et de sentiment sur ces problèmes."

Les cultures vont prendre une grande importance dans les formations au changement. Nous avons retenu trois aspects des cultures:

a) La culture de l'entreprise CVMM dont nous rappelons ici une phrase clé: "think globally, act locally".

Cette culture serait pour Schein du niveau des valeurs et nous lui associerons le niveau 3 c'est à dire celui des comportements observables.

b) La culture "Leading Business Schools", qui est en fait une transculture commune à tous les dirigeants des filiales et à ceux du siège de CVMM Europe.

Cette culture est généralement à dominante américaine et forgée par la lecture d'ouvrages sur le management et la stratégie. Elle est régulièrement entretenue par des séminaires internationaux ; le modèle américain a longtemps été le modèle de référence en matière de management.

Il le reste encore pour une large part et constitue souvent une norme, un standard international. Il a été diffusé par la pratique dans l'entreprise, mais aussi par les ouvrages des auteurs qui l'ont étudié (de P. Drucker à Peters et Watermans) et par l'influence des "Business Schools" chargées des programmes de formation continue ainsi que des grands cabinets de conseil (Mc Kinsey, Boston Consulting Group, Arthur Andersen, etc.). 
c) Enfin, les cultures locales, toutes spécifiques, et dont les pratiques managériales sont très différentes. Ces différences s'expriment notamment dans la relation à l'autorité, au temps, à la prise de décisions ou aux modes mêmes de travail.

L'Europe est un complexe (complexus, ce qui est tissé ensemble) dont le propre est d'assembler sans les confondre les plus grandes diversités et d'associer les contraires de façon non séparable?

Dans cette étude des différents types de culture, nous nous servirons d'un tableau issu de l'ouvrage de référence de F. Trompenaars, Riding the Waves of Culture.

Ce tableau a été obtenu par la compilation de 3000 cas, principalement des cadres, et donne la représentation suivante:

\section{CULTURES ET STYLES DE MANAGEMENT ${ }^{10}$}

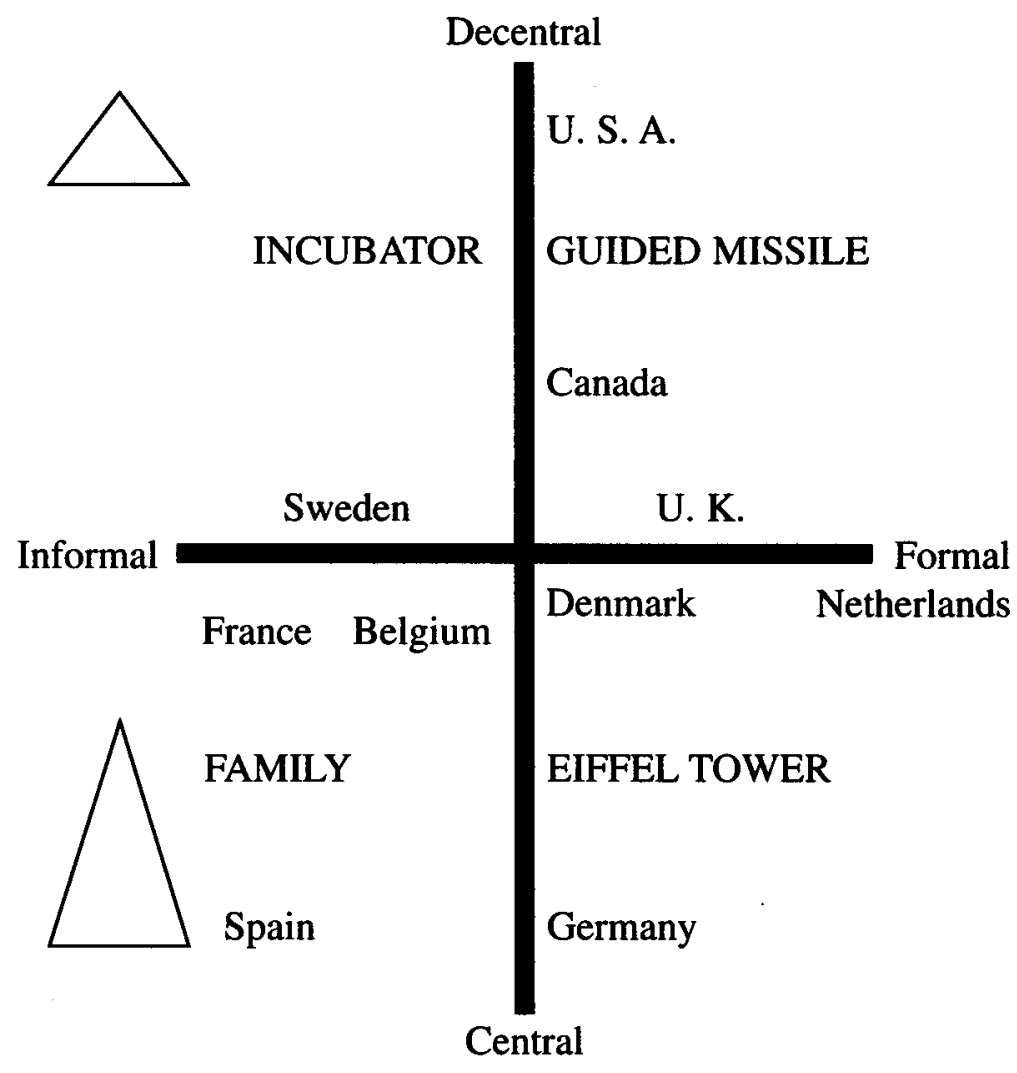


Ce tableau va nous permettre de suivre plus aisément les adaptations que feront les acteurs de la formation pour rendre possible et transmettre cette conduite du changement. Si les requêtes initiales des Directeurs Généraux des filiales étaient identiques dans leur formulation, elles ne recouvraient pas la même réalité et nécessitaient un transcodage.

Les cadres intermédiaires, tant locaux que ceux du siège, effectuèrent un constant travail de modulation/démodulation des messages et des actions. Comme l'ont montré $\mathrm{D}$. Bollinger et $\mathrm{G}$. Hofstede, la relation des individus dans l'organisation est fortement liée à leur capacité à supporter l'incertitude, mais aussi à la nature de la relation que ces acteurs entretiennent vis-à-vis de la hiérarchie. $\mathrm{Si}$, en France comme en Italie, la distance à la hiérarchie est grande et si la nature du rapport entre les individus se situe dans une logique de l'honneur qui révèle, comme l'écrit P. Irribarne: "l'existence des devoirs de chaque état pour définir ce qu'il considère comme honorable ou contraire à l'honneur", il n'en est pas de même en Allemagne ou en Scandinavie. En Italie, dans les entreprises, la relation est souvent de type familial avec un père extrêmement présent dans la représentation de la fonction managériale, le comportement des acteurs étant régi par un code de la famille. En Allemagne, la situation est différente dans un contexte culturel qui, s'il favorise l'échange entre acteurs, le situe dans un contexte organisationnel dont les règles et les procédures sont définies, bien que dans l'organisation la distance hiérarchique soit plus faible qu'en Italie.

\section{L'ALLEMAGNE: UNE FORMATION GÉNÉRALISÉE DANS UN CON- TEXTE DE CULTURE EXPLICITE}

Le transfert des programmes de formation s'est effectué directement entre la Grande-Bretagne et l'Allemagne sous l'impulsion initiale du Directeur Général allemand qui avait donné une priorité absolue à cette opération. En conséquence, l'équipe anglaise, "le chef de projet" et les cadres associés ayant reçu "leur autorité" du Directeur Général, purent transmettre à la ligne hiérarchique les contenus des formations; le programme fut piloté par une équipe de cadres, équipe dédiée qui travailla indépendamment des structures "normales" de formation, ce qui était logique, car cette action ne s'inscrivait pas dans les plans de développement prévus par la direction de la formation.

Parallèlement, l'Allemagne mit en place une organisation matricielle. Force est de constater que l'appropriation de nouvelles façons de travailler et d'un nouveau mode relationnel ne s'est pas faite d'une façon globalement satisfaisante. Là où l'équipe anglaise parlait "équipe", il fut compris "groupe". Ces distinctions sont expliquées dans l'article: Why teams matter? 
If teams provide such a critical level of performance why is it that so many managers remain confused about what -exactly- they are? Some mentally lump them together with tasks forces, committees, departments and other forms of groups

Others think of them not in organisational terms but as the emboissement of values as TEAMWORK or cooperation or empowerment. Still others believe that merely calling a group a team makes it one. It does not".

Les auteurs définissent l'équipe comme étant constituée d'un petit nombre de personnes dont les compétences sont complémentaires, partageant un objectif commun et un niveau de performance à atteindre. Il est d'autre part nécessaire de définir un mode de travail en commun avec une responsabilité globale auto-allouée.

Dans ce transfert le cadre opérationnel allemand n'avait pas perçu et ne s'était pas approprié le projet ; il était responsable devant une hiérarchie de la diffusion d'une action décidée par son directeur général et il se contentait d'appliquer et de transmettre une nouvelle règle, selon le principe de la "réduction de la dissonance" afin de rapprocher le nouveau mode de relation et de travail souhaités d'un mode connu par lui et accepté par ses collaborateurs.

H. LANDIER décrit parfaitement le dilemme devant lequel se trouvait le cadre intermédiaire allemand :

Le changement étant un processus à la fois collectif et créatif, il échappe à ceux qui se trouvent à son origine. Il compte une large part d'imprévu. En outre, s'il est irréductible à un ensemble de recettes qu'il suffirait d'appliquer pour être assuré du succès, celui-ci implique toutefois que soient réunies certaines conditions préalables ${ }^{12}$.

Sa culture lui dictait son attitude vis-à-vis de l'autorité, sa tolérance pour le mode de changement tel qu'il était conçu par l'Angleterre était limitée. Il y eut donc une perte d'efficacité. Le transcodage culturel n'avait pas pu être pris en compte. Le mode de transfert choisi avait malheureusement ignoré un certain nombre de réalités fortes. La première de ces réalités est que le mode de fonctionnement d'une organisation repose sur une codification élaborée des procédures ainsi que sur une compétence à priori reconnue et associée des différentes fonctions. Le fait de ne pas utiliser les ressources de la formation interne créait une situation perçue comme ne respectant pas l'usage, ce qui, au-delà d'une faute de goût, eut une conséquence directe: "wenn Training nicht durch professionelle Trainer durchgeführt wird, liegt das daran, dass Paris am Program gewerkelt hat"l3.

La deuxième erreur fut d'introduire un mode de fonctionnement matriciel sans modifier avant les règles et de s'en remettre aux acteurs pour ajuster dans la pratique et le vécu l'organisation du travail. Le programme visant à modifier "l'espace où s'articulent et se coordonnent ses différentes fonctions, ses différentes activités" ainsi que les "modes de coopération de ses membres". Par contre, 
cette approche fit l'impasse sur la nature des rapports sociaux en particulier sur les modes de mobilisation des salariés. Comme le souligne D. Linhart, l'asynchronisme entre ces trois composantes risque de produire un modèle bancal, et ce d'autant plus que les cultures anglaises, françaises et allemandes ont des approches et des pratiques différentes de la relation sociale et contractuelle. Le contenu de ces formations montra encore des différences notables, telles qu'absences de références crédibles pour les populations cibles, mises en avant de situations favorisant la prise de décisions dans un environnement ambigu et enfin l'utilisation de jeux (LEGO) destinés à mettre en évidence certains modes de fonctionnement. Ces activités étaient placées sous la responsabilité de l'encadrement qui avait la charge d'interpréter les zones floues.

\section{L'ITALIE: UNE FORMATION GÉNÉRALISÉE DANS UN CONTEXTE DE CULTURE IMPLICITE}

L'approche italienne nous offre une déclinaison différente dans la généralisation de l'expérience anglaise.

Le responsable du développement des ventes et le responsable de la formation, un cadre intermédiaire expérimenté, décidèrent qu'il serait important que l'Italie mette en oeuvre un tel processus de changement. A partir de ce moment, le projet fut mené par des cadres intermédiaires. Une invitation de la part du siège de CVMM Europe à quelques cadres intermédiaires italiens, agents d'influence et/ou responsables de liaison avec le siège (marketing/développement des ventes /formation) réactiva un tissu relationnel préexistant. Le projet passa par les phases suivantes:

- rencontre en Italie pour débattre de l'intérêt éventuel d'une telle expérience;

- invitation à assister à un des événements en Grande-Bretagne;

- prise en charge en Grande-Bretagne par le responsable CVMM Europe des invités italiens;

- visite et réunions à Milan pour analyser les plus et les moins de l'expérience anglaise;

Suivit une étape très importante, la temporisation de l'action qui permit :

- A l'Italie de décliner un modèle "compatible mais italien" du processus de changement incluant les particularités de l'organisation locale et des modes de fonctionnement effectifs. 
- Aux responsables du siège de CVMM Europe de trouver auprès du Comité Directeur un parrain, un champion, pour cette action et de lancer une valorisation à priori de l'effort entrepris.

- La transposition et la traduction de l'ensemble des supports et outils en synergies, ce qui permit les adaptations nécessaires à la culture locale.

- Le maillage très étroit de l'organisation par les cadres intermédiaires du siège pour sécuriser leurs vis-à-vis italiens et leur faire obtenir l'approbation de la Direction Générale, qui, à son tour, "lança" le projet déjà avancé, évitant ainsi des risques.

Nous pouvons dire qu'un travail de "Sherpa" fut effectué, destiné à préparer les conditions nécessaires à l'acceptabilité d'un tel processus. Il paraît important de noter également que ceci fut favorisé par la pratique de l'italien par l'ensemble des acteurs, par une "dramatisation" de la relation à l'autorité formelle et par une implication progressive des différents échelons hiérarchiques à mesure que le risque diminuait. Le résultat fut extrêmement positif. Il permit en effet d'aborder la réflexion sur les structures opérantes, les modèles de distribution, et les limites des territoires personnels.

Enseignements: le succès du transfert par le maillage :

L'action globale favorisa la remise en cause progressive des modes de fonctionnement. En effet, le mode de management dominant en Italie était d'un type traditionnel, proche de la famille ou du clan ; les décisions reposaient plus sur un réseau d'obligations mutuelles et d'interdépendances que sur une simple réalité économique. La prise en compte par les acteurs du siège de CVMM Europe de la réalité culturelle italienne et un mode de dialogue fondé sur la persuasion et l'implicite, ont permis l'amorce du changement.

Le suivi, essentiel pour le succès d'une telle opération, fut assuré par un maillage étroit des fonctions entre les mains de l'encadrement intermédiaire. Le succès de ce transfert reposa sur la capacité à gérer l'ambigu dont firent preuve les cadres intermédiaires, qu'ils soient responsables de formation ou responsables opérationnels. Ils amorcèrent un processus les dépassant globalement sans menacer la stratégie dirigeante de CVMM Italie et en donnant à leurs vis-à-vis cadres locaux l'occasion d'asseoir pour un temps leur pouvoir. Les acteurs de CVMM Europe et d'Italie avaient mis en pratique une théorie ancienne de Tuckman $^{14}$ : 


\section{DEVELOPMENTAL SEQUENCES IN SMALL GROUPS}

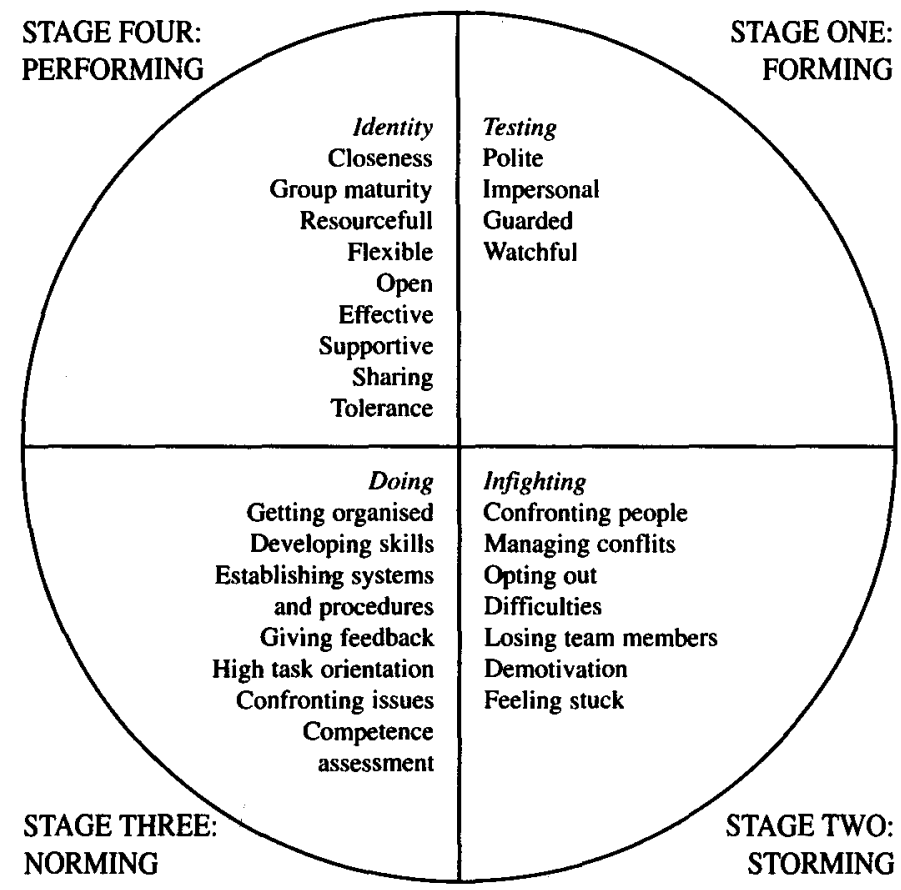

Et comme l'écrit le professeur A. Kakabadse:

Simply placing a number of skilled professional individuals in a group and expecting them to perform as a team is unrealistic ; for teams to perform reasonably takes time: time for people to become better acquainted with each other to assess each other's strengths and weaknesses $^{15}$.

L'amorce du changement par le programme TEAMWORK allait se généraliser en Italie. La réforme organisationnelle fut postérieure. Si nous nous référons à notre tableau, nous pouvons dire que la prise en compte de la dimension "familiale" de l'enjeu par des cadres intermédiaires dans la définition et la transmission du programme de formation assura le succès là où une pure vision "Business School" américaine aurait conduit à l'échec. C'est par un respect de la culture locale et surtout de la culture vécue plutôt que dite, par l'acceptation a priori de différences, la maîtrise de la langue et enfin la capacité à vivre l'incertain et l'ambigu que ce transfert fut un succès culturel et non un succès de l'impérialisme organisationnel. 


\section{LA SCANDINAVIE: LA FORMATION GÉNÉRALISÉE SANS IMPLICA- TION DE L'ENCADREMENT INTERMÉDIAIRE}

L'organisation scandinave, née d'une réorganisation antérieure, fonctionne sur un mode matriciel avec un Comité Exécutif dont la particularité est d'être composée pour trois des fonctions-clés d'un Anglais (Directeur Général) et de deux Français (Développement des ventes et Service Client), d'avoir son siège en Suède avec un Directeur des Ressources Humaines et un Vice Président norvégiens (lui-même ancien Directeur Général de la Norvège).

Le mode de fonctionnement matriciel théorique est biaisé chaque jour afin de prendre en compte les réalités culturelles de chacun des pays. Il semble quasiment impossible à un client danois de dépendre d'un responsable de compte norvégien et cela devient proprement inconcevable si ce responsable était suédois. D'où un mode de "dysfonctionnement" parallèle, auquel le Comité Exécutif souhaita mettre fin.

L'adhésion au programme anglais était la solution, et l'accord se fit entre le Développement des Ventes de la Scandinavie et les Ressources Humaines du siège de CVMM Europe à la lisière d'une réunion. L'adhésion de la Direction Générale était acquise, celle du siège aussi, ce qui permit un portage des programmes pour répondre aux besoins locaux de changement (besoins exprimés par la Direction Générale, mais fortement refusés par la hiérarchie en place).

La mise en oeuvre du transfert se fit majoritairement en utilisant des ressources externes à l'entreprise, équipes de formation fortement motivées, mais tout aussi ignorantes de la réalité organisationnelle et culturelle des pays concernés. Cette équipe alla jusqu'à concevoir les discours des Directeurs Généraux de chacun des pays. Le déroulement du programme servit à cristalliser les conflits latents et le mal-être de l'ensemble des membres de l'organisation, en particulier en Norvège, où ils rejetèrent l'ensemble du programme comme ne correspondant pas à un besoin réel. Ils justifièrent leur refus par le fait qu'ils étaient la première filiale CVMM à être certifiée ISO 9000 et n'avaient donc pas de raison de changer.

Il fallut plusieurs mois de visites et d'interventions de la Direction Formation de CVMM Europe pour comprendre le rejet de ces programmes de changement.

Les enseignements d'un échec :

Le programme a échoué principalement pour deux raisons :

Il apparut clairement que la non-implication de l'encadrement local dans le processus de transmission des formations au changement avait été une faute majeure. D'autre part, la projection d'un modèle anglais accepté par le Direc- 
teur Général anglais, heurtait profondément la sensibilité locale et allait à l'encontre de ce que définissait $F$. Trompenaars:

An incubation de culture is based on the existential idea that organisations are secondary to the fulfilment of individuals. If organisations are to be tolerated at all, they should be to serve there, to serve as incubations for elf expression and self fulfilment ${ }^{16}$.

Ces programmes de changement permirent à la Direction des Ressources Humaines de CVMM Europe d'analyser la tolérance limite d'un système (la Scandinavie avait en effet perdu $30 \%$ de ses effectifs en un an), de mesurer l'importance d'un relais formé par l'encadrement intermédiaire et de la présence d'une équipe de formation locale pour atteindre les objectifs d'une Direction Générale. Il semblait difficile de court-circuiter les couches de cadres locaux pour s'adresser efficacement à la base dès lors qu'il s'agissait de relever des enjeux primordiaux pour l'entreprise. Une des conditions de la réussite de la conduite du changement était d'impliquer fortement ceux qui étaient les plus concernés: les cadres intermédiaires, tant du côté des équipes support que des équipes locales.

L'illusion générée par l'anglais, langue parfaitement parlée localement, et une similitude de culture, furent fatales à cette formation. II fallut admettre que l'on ne change pas son comportement dans une langue de transfert, sauf si l'on est seul parmi d'autres étrangers et que cette langue est la seule passerelle commune.

Nous pûmes vérifier empiriquement le poids des cultures et leur capacité à résister à des réorganisations qui semblent logiques.

Suite à cette expérience, il fut décidé que la Direction des Ressources Humaines conduirait en langue locale un programme d'évaluation, assessment seminar, qui permettrait aux différents cadres intermédiaires de faire un bilan de leurs forces et faiblesses, de construire leur programme de développement personnel et sur cette base, de conduire un travail de fond sur le changement nécessaire à l'organisation. Ce programme est en cours.

\section{CONCLUSION: CULTURES, ACTEURS ET CONDUITE DU CHANGE- MENT EN EUROPE}

Notre exemple met en évidence les rapports existant entre les différent types de culture et l'implication des acteurs. Nous souhaiterions en tirer des enseignements utiles à la conduite d'actions de changement en environnement interculturel.

Le schéma ci-après permet aux acteurs concernés par la conduite du changement d'anticiper les réactions possibles et d'adapter une série de mesures desti- 
nées à compenser les effets de résistance ou à adopter une technique différente pour faire changer l'organisation.

\section{CULTURE ET IMPLICATION}

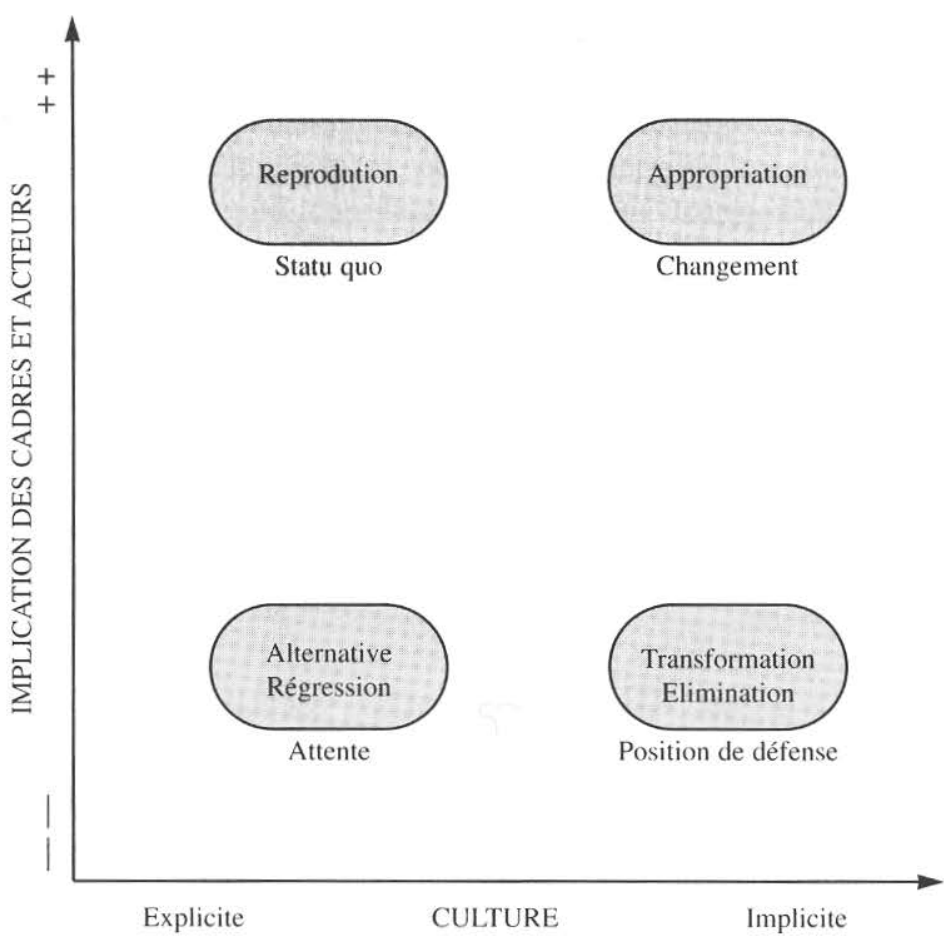

Une culture explicite, alliée à une non implication des acteurs, et notamment de l'encadrement intermédiaire, génère une situation de perte de temps, de consommation maximale, de ressources annexes destinées à stopper, ralentir ou dévier le projet en jouant sur le principe suivant: "ils" changeront de projet tôt ou tard, alors il suffit d'attendre ou d'appliquer à minima. Les effets sont globalement désastreux sur les populations concernées. La nature des relations avec le siège, donc avec d'autres cultures, sert généralement d'exutoire aux employés trahis.

Le deuxième risque se présente dans le cas d'une culture explicite avec implication de l'encadrement intermédiaire. Dans ce cas, il s'agit de bien faire plutôt que de faire bien. Une telle situation entraîne une reproduction à l'identique du modèle de référence empreint d'une culture différente. Dans notre exemple un 
programme destiné à favoriser le travail d'équipe Teamwork devient un programme qui favorise la constitution d'équipe, Teambuilding. En effet, la culture $s$ 'attache à développer un existant, par exemple le travail en groupe structuré. Elle renforce les liens à l'intérieur du groupe plutôt qu'elle ne développe une capacité à se reconfigurer en fonction d'un projet, objectif que vise l'organisation. L'implication ne suffit pas à assurer la mise en oeuvre du changement, sinon d'une façon limitée.

Un autre risque, de nature différente, se rencontre quand des actions sont mises en oeuvre sans que l'encadrement intermédiaire soit impliqué et dans un contexte de culture implicite. La non implication de l'encadrement des acteurs locaux va entraîner des actions cosmétiques dont le seul but est de montrer l'inadaptation du programme à la situation locale par une déformation plus ou moins grande du contenu et des processus. Nous pouvons avoir utilisation du nom du programme avec un contenu totalement différent, ou même réutilisation d'anciens programmes sous le nouveau nom. Les résultats seront généralement là encore désastreux. Cette situation aura renforcé une position de défiance vis-à-vis du changement. Elle risque de se retrouver dans des pays comme l'Espagne, si les acteurs ne voient pas comment il vont sauvegarder leurs acquis devant les modifications demandées. Il faudra prendre des décisions, trancher, remplacer des acteurs ou modifier les structures de façon importante.

Enfin, le dernier cas, celui d'une implication des cadres, des acteurs locaux dans un contexte de culture implicite est la condition optimum du succès. En effet, dans ce cas de figure, que nous avons rencontré en Italie, l'expérience du changement devient une réalité organisationnelle et opérationnelle. C'est une situation idéale qui permet seulement alors de vérifier la réalité de: "Think globally Act locally".

\section{NOTES}

I TOCQUEVILLE, A. de, De la Démocratie en Amérique, GF-Flammarion, Paris, 1981, p. 528 , tome 1 .

${ }^{2}$ Deysine A., Teule-Martin C., Réussir sur le marché américain, Jupiter, Paris, 1989, p. 13.

${ }^{3}$ SPOTLIGHT (projecteur) est un ensemble de programmes de formation-action.

${ }^{4}$ Rapport BAIN, Septembre 1991, Company confidential.

${ }^{5}$ Rapport BAIN, Septembre 1991, Company confidential.

${ }^{6}$ Cluster $=$ regroupement de plusieurs pays voisins dans le but d'optimiser le coût de fonctionnement. 
$7++=$ bilingue

$++=$ excellente maitrise(écrit et oral)

$+/-=$ maîtrise orale

- - = non maitrîse de la langue

${ }^{8}$ HAMPDEN-TURNER, C., La culture d'entreprise, Seuil,Paris, 1992, p. 24.

${ }^{9}$ MORIN E., Penser l'Europe, Paris, Gallimard, 1987, in Pratique du Management en Europe, op-cit., p. 214.

${ }^{10}$ D'après: TROMPENAARS F., Riding the Waves of Culture, London, The Economist Book, 1993, P.161.

" KATZENBACH J. R., SMITH D.K., "Why Teams matter", The McKinsey Quarterly, 1992, n. $^{\circ} 3$.

${ }^{12}$ LANDIER H., L'entreprise intelligente, Calmann-Levy, Paris, 1991, p. 72.

${ }^{13}$ traduction française: "si ce n'est pas un formateur qui vient nous faire cours, alors c'est encore une idée bricolée du siège français".

14 TUCKMANN B.W., "Developmental sequences in small groups", Psychological Bulletin, 1965; Vol. 63, n. ${ }^{\circ} 6$.

15 KAKABADSE A., Working in organizations, Penguin Books, London, 1988, p. 358. ${ }^{16}$ TROMPENAARS F., op-cit., p.. 157.

\section{BIBLIOGRAPHIE}

AUBREY, B. (1990), Savoir faire savoir, InterEditions, Paris.

CARLZON, J. (1986), Renversons la pyramide, InterEditions, Paris.

CHAIZE, J. (1992), La porte du changement s'ouvre de l'intérieur, Éditions Calmann-Lévy, Paris.

CROZIER, M. (1991), L'entreprise à l'écoute, InterEditions, Paris.

DE BOISANGER, P. (octobre 1990), "Le management en univers instable", Revue Française de gestion, $\mathrm{n} .{ }^{\circ} 80$.

DEYSINE, A., TEULE-MARTIN, C. (1989), Réussir sur le marché américain, Jupiter, Paris. HAMPDEN-TURNER, C. (1992), La culture d'entreprise, Seuil, Paris.

HOFSTEDE, M. G., et BOLLINGER, D. (1987), Les différences culturelles dans le Management, Les Editions d'organisation, Paris.

KAKABADSE, A. (1991), The Wealth Creators, Kogan Page Ltd, London.

KAKABADSE, A. (1987), Working in organizations, Penguin Books, London.

LANDIER, H. (1992), L'entreprise intelligente, Ville d'Avray, Pygmalion Records (Conférence).

LANDIER, H. (1991), Vers l'entreprise intelligente, Calmann-Lévy, Paris.

LINHART, D. (1994), La modernisation des entreprises, La découverte, Paris.

LIPOVETSKI, G. (1991), "Les noces de l'éthique et du business", Le débat, n. ${ }^{\circ} 67$.

MORIN, E. (1987), Penser l'Europe , Gallimard, Paris. 
PAIN, A. (1992), Évaluer les actions de formation, Les Éditions d'organisation, Paris.

SIEVERS, B. (1990), "la motivation: un ersatz de signification", L'individu dans l'organisation. Les Presses de l'Université Laval, Québec.

SIMONET, J. (1992), Pratiques du Management en Europe, Les Editions d'organisation, Paris. TOCQUEVILlE, A. de (1981), De la Démocratie en Amérique, GF - Flammarion, Paris.

TROMPENAARS, F. (1993), Riding the waves of culture, The Economist, Books, London. TRUC, R. (1991), Former pour des résultats, Ressources Humaines et Management, ESF, Paris. USINIER, J. C., EASTERBY-SMITH, M., THORPE, R. (1993), Introduction à la recherche en gestion, Paris, Gestion Economica.

WATZLAWICK, P. (1980), Le langage du changement, Le Seuil, Paris. 